\title{
Numerical algorithm improvements for the CFD- code ANES for turbulent flow simulations via the LES method
}

\author{
Valery Artemov ${ }^{1}$, Maksim Makarov ${ }^{1 *}$, Georgiy Yankov ${ }^{1}$ and Konstantin Minko ${ }^{1}$ \\ ${ }^{1}$ National Research University «Moscow Power Engineering Institute», Moscow, Russia
}

\begin{abstract}
Turbulent flow simulations via the LES method place high demands on the accuracy of the utilised numerical schemes. This work considers issues of both the implementation and improvement of numerical schemes and algorithms in the 'in-house' CFD code ANES [1]. A new second-order scheme (MAK) was developed by the authors. Its accuracy was studied by solving well-known test problems, with the aim of extending its use to complex turbulent flow calculations.
\end{abstract}

\section{Numerical algorithms}

The ANES code uses a control volume (CV) method. Both structural and non-structural grids with cut-cells and the fine grid algorithm are implemented in the code.

Three well-known and widely used methods of second-order accuracy in space $[2,3,4]$ - QUICK, MUSCL and SuperBEE - are also implemented in the ANES code. A primary feature of these algorithms' application for the CV method is using the gradient of the unknown function at the $\mathrm{CV}$ nodal points to calculate the total flux of that function through the CV face. The gradient of the function is calculated via two algorithms: 1) the leastsquares method with a Gaussian weight model and 2) using interpolation of the function values on the $\mathrm{CV}$ face and the gradient computation based on the Ostrogradsky-Gauss theorem.

The authors have proposed an original scheme called MAK for calculating the total flux of the $\Phi$-function on the CV face based on the exact solutions of a one-dimensional steady equation with convective, diffusive and source terms and constant coefficients:

$$
\frac{\mathrm{d}}{\mathrm{d} x}\left(\rho u \Phi-\Gamma \frac{\mathrm{d} \Phi}{\mathrm{d} x}\right)=S
$$

The analytical solution of equation (1) on the segment that connects the nodal points $P$ and $E$ of two adjacent CVs with the boundary conditions $\Phi\left(x_{E}\right)=\Phi_{E}$ and $\Phi\left(x_{P}\right)=\Phi_{P}$ is well known. In the multidimensional formulation of the problem, the value of $S$ will contain fluxes in different directions from the $x$-direction. To find this value, a linear combination

* Corresponding author: makarovmv2000@yandex.ru 
of derivatives at the nodal points $G_{P}=\left.(\mathrm{d} \Phi / \mathrm{d} X)\right|_{X=0}$ and $G_{E}=\left.(\mathrm{d} \Phi / \mathrm{dX})\right|_{X=1}$ was used. The result was an exact expression of the density of total flow through the common face 'e' of two $\mathrm{CVs}$ with nodal points $P$ and $E$ :

$$
J_{e}=\left(\rho u \Phi-\Gamma \frac{\mathrm{d} \Phi}{\mathrm{d} x}\right)_{e}=m \Phi_{P}+A d\left(\Phi_{P}-\Phi_{E}\right)+\left(X_{e}-\frac{1-A}{P}\right)\left(m G_{P}+A d\left(G_{P}-G_{E}\right)\right)
$$

where $X=\left(x-x_{P}\right) / \delta, \delta=x_{E}-x_{P}, m=(\rho u)_{e}, d=\Gamma / \delta, P=m / d, A=\frac{P}{\exp (P)-1}$.

As for time integration algorithm, the second-order, three-level implicit scheme, which is called the Second Order Backward Euler Scheme (SOBES), was implemented in the ANES code.

To calculate the pressure field in the ANES code, a modified version of the SIMPLE + PISO algorithm was used for both structured and unstructured grids, with the step time restricted by the Courant number.

\section{Verification of numerical algorithms and schemes}

In the first test, a steady flow at an angle of $45^{0}$ to the grid lines was considered. The flow with a density of $\rho=1$ in a $2 \mathrm{D}$ square domain with dimensions $L_{x}=L_{y}=1$ and components of the velocity $u_{x}=u_{y}=1$ was investigated. The equation for the scalar function $\Phi$ was solved. The boundary conditions for this equation were as follows: $\Phi=1$ for the 'left' input boundary $(x=0)$ and $\Phi=0$ for the 'down' input boundary $(y=0)$. Unlike [5], a small diffusion coefficient in the $\Phi$-equation (Peclet number $=1000$ ) was included for testing of a common influence of both convection and diffusion.

The 'exact' solution was obtained on a $1000 \times 1000$ grid. The main calculations were carried out on the $64 \times 64$ grid for various schemes. The results indicated that the scheme of the first-order POWER [5] had a high scheme diffusion. The scheme of the second-order SuperBEE gave more accurate results; however, maximum accuracy was obtained when using QUICK, MUSCL and MAK schemes.

The second test was a classical two-dimensional problem of flow in a square cavity with a moving wall at $\mathrm{Re}=1000$. There is a 'reference' solution for this task [6]. The calculations used the unstructured mesh, with $64 \times 64$ nodes. QUICK, MUSCL and MAK schemes showed the best results.

The next problem - the attenuation of two-dimensional vortices of Taylor-Green [7] was considered. The computational domain was a square with the dimensions of $2 \pi L \times 2 \pi L$. The periodic boundary conditions were imposed at the opposite boundaries.
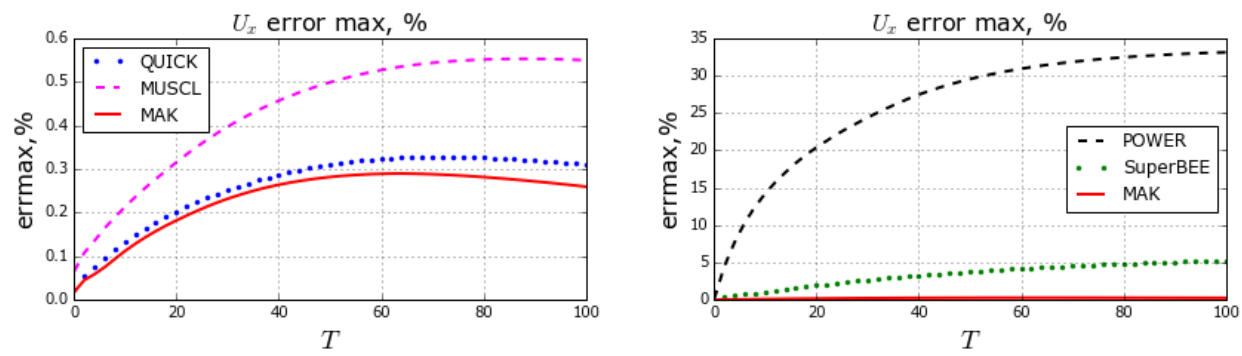

Fig. 1. The maximum error of velocity $U_{x}$ for different schemes.

The 2D Navier-Stokes equations for this problem have the exact solution of 


$$
U_{x}=\frac{u_{x}}{U_{0}}=\sin (X) \cdot \cos (Y) \cdot \exp \left(-\frac{2 T}{\mathrm{Re}}\right), \quad U_{y}=\frac{u_{y}}{U_{0}}=-\cos (X) \cdot \sin (Y) \cdot \exp \left(-\frac{2 T}{\mathrm{Re}}\right)
$$

where $X=x / L=0 . .2 \pi, \quad Y=y / L=0 . .2 \pi, \quad \operatorname{Re}=U_{0} L / v, \quad T=t U_{0} / L-$ dimensionless time, $v$ - kinematic viscosity.

The calculations were performed at $\mathrm{Re}=100$ on the uniform $64 \times 64$ grid; the step time was 0.05 . For integrating in time, we used the second-order SOBES scheme. Fig. 1 shows the maximum estimated error of the velocity $U_{x}$, which was normalized by the value $\exp (-2 T / \mathrm{Re})$. The maximum solution accuracy was obtained by using the MAK scheme.

The calculations of the Taylor-Green vortex flow were performed in a threedimensional formulation for $\mathrm{Re}=1600$, which corresponded to the mode of decay of the isotropic turbulence. The Smagorinsky model for subgrid turbulent viscosity with $C_{S}=0.2$ was used on the $192 \times 192 \times 192$ grid with a step time of 0.005 .

Fig. 2 shows the results of calculations using the SuperBEE schemes and SOBES in comparison with the DNS data [8].
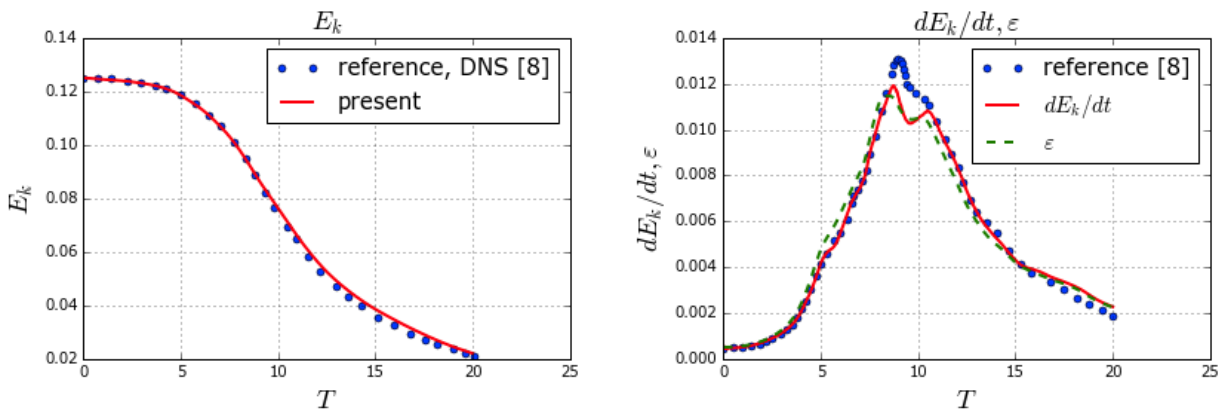

Fig. 2. The kinetic energy $E_{k}$ and its rate of dissipation $\varepsilon$.

The results of modelling a steady flow in a flat channel for conditions that correspond to the work of R. Moser, J. Kim and N. Mansour (MKM) [9] are shown in Fig. 3.
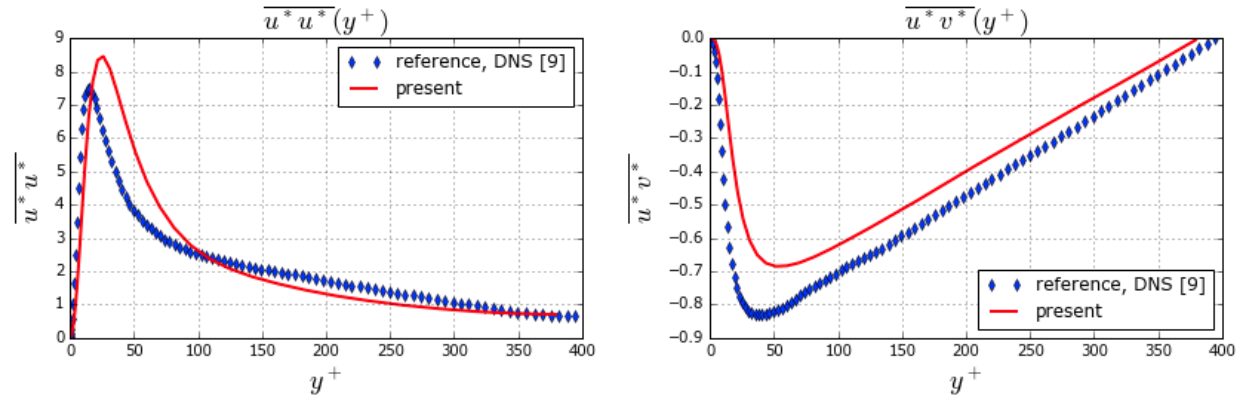

Fig. 3. The intensity of fluctuations of the $x$-component of the velocity and the LES component of the turbulent shear stress, $\operatorname{Re}_{\tau}=395$.

Calculations of the lifting, fully developed turbulent flow with buoyancy forces in a vertical plane channel and a width of $2 \delta$ for the conditions of [10] were carried out. On the channel walls, constant temperatures were kept. For the right cold wall $\theta(y=2 \delta)=\theta_{c}$, and for left hot wall $\theta(y=0)=\theta_{c}+\Delta \theta$. The following dimensionless values were used:

$$
u_{\tau}^{*}=\sqrt{\tau_{w} / \rho}, U^{*}=u / u_{\tau}^{*}, \Theta=\left(\theta-\theta_{c}\right) / \Delta \theta, \operatorname{Re}_{\tau}=u_{\tau}^{*} \delta / v, \text { Gr }=g \beta \Delta \theta(2 \delta)^{3} / v^{2}
$$


where $\tau_{w}$ - the wall shear-stress averaged on the two walls, $g$ - acceleration of gravity and $\beta$ - the coefficient of volume expansion.

By integrating the streamwise mean momentum equation with respect to $\mathrm{Y}$, one can obtain the following balance equation:

$$
\tau^{*}(0)-Y=\frac{1}{\operatorname{Re}_{\tau}} \frac{\mathrm{d} U_{x}^{*}}{\mathrm{~d} Y}-\overline{u^{*} v^{*}}+\frac{\mathrm{Gr}}{\operatorname{Re}_{\tau}^{2}} \int_{0}^{Y}\left(\Theta-\Theta_{m}\right) \mathrm{d} Y, Y=y / \delta, \tau^{*}(0)=\frac{\tau(Y=0)}{\tau_{w}}
$$

where $\overline{u^{*} v^{*}}$ - dimensionless Reynolds stress and $\Theta_{m}$ - arithmetic mean dimensionless temperature over the channel cross section.

The calculations were performed using Boussinesq approximation via the MAK scheme on a $64 \times 32 \times 32$ grid. The results were compared with the DNS modelling of [10] (Fig. 4).
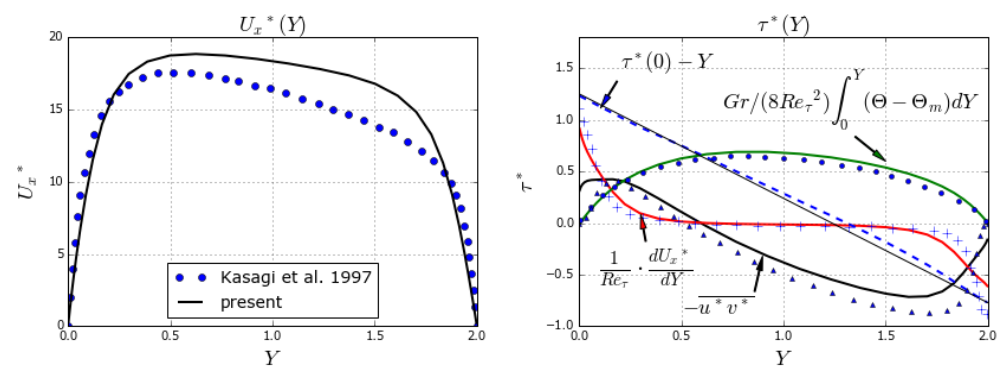

Fig. 4. Dimensionless mean velocity and stresses profiles for $\operatorname{Re}_{\tau}=150$ and $\mathrm{Gr}=9 \cdot 6 \cdot 10^{5}$. Markers indicate the DNS data [10].

\section{Conclusion}

The performances of various schemes were analysed via the LES method with a simple subgrid turbulent viscosity of the Smagorinsky model on the coarse grids. The results showed that the proposed MAK scheme worked efficiently for a wide range of problems, which allowed its use with the LES method for complex turbulent flow.

The authors extend sincere thanks for the financial support of the Russian Foundation for Basic Research (project № 16-08-00981-a, №17-08-01007-a).

\section{References}

1. http://anes.ch12655.tmweb.ru

2. B.P. Leonard, Comp. Methods Appl. Mech. 1959 (1979)

3. P. K. Sweby, SIAM Journal on Numerical Analysis, 21, 5 (1984)

4. Ferziger J.H., Peric M. Computational methods for fluid dynamics (Springer, 2002)

5. S. Patankar, Numerical heat transfer and fluid flow (Hemisphere, 1980)

6. U. Ghia, K.N. Ghia, C.T. Shin. J. Comp. Phys. 48, 387 (1982)

7. G. I. Taylor and A. E. Green, Royal Society of London Proc. Ser. A, 158895 (1937)

8. http://www.as.dlr.de

9. R.D. Moser, J. Kim, N.N. Mansour, Phys. Fluids., 11, 943 (1999)

10. N. Kasagi, M. Nishimura, Int. J. Heat Fluid Flow, 18, 88 (1997) 